\title{
Comparative long-term outcomes for pancreatic volume change, nutritional status, and incidence of new-onset diabetes between pancreatogastrostomy and pancreatojejunostomy after pancreaticoduodenectomy
}

\author{
Bong Jun Kwak, Ho Joong Choi, Young Kyoung You, Dong Goo Kim, Tae Ho Hong \\ Department of Hepatobiliary and Pancreas Surgery, Seoul St. Mary's Hospital, College of Medicine, The Catholic University of Korea, Seoul, \\ Republic of Korea \\ Contributions: (I) Conception and design: TH Hong; (II) Administrative support: DG Kim; (III) Provision of study material or patients: YK You, DG \\ Kim, TH Hong; (IV) Collection and assembly of data: HJ Choi; (V) Data analysis and interpretation: BJ Kwak, TH Hong; (VI) Manuscript writing: \\ All authors; (VII) Final approval of manuscript: All authors. \\ Correspondence to: Tae Ho Hong. Department of Hepatobiliary and Pancreas Surgery, Seoul St. Mary's Hospital, College of Medicine, The Catholic \\ University of Korea, 222, Banpo-daero, Seocho-gu, Seoul, 06591, Republic of Korea. Email: gshth@catholic.ac.kr.
}

Background: The difference in volume change in a pancreatic remnant according to the type of pancreaticoenterostomy after pancreaticoduodenectomy (PD) for long-term follow-up is unknown. Also, there are few studies that evaluate the difference in general nutritional status and pancreatic endocrine function, including new-onset diabetes mellitus (NODM) depending on the type of pancreaticoenterostomy. This study aimed to compare serial pancreatic volume changes in pancreatic remnants between pancreatogastrostomy (PG) and pancreatojejunostomy (PJ) after PD and to evaluate the difference in general nutritional status and incidence of NODM between PG and PJ.

Methods: This study enrolled 115 patients who had survived for more than 3 years after PD. They were divided into the PG group and the PJ group. Their clinicopathologic factors were collected and analyzed. We calculated serial pancreas volume and pancreatic duct size precisely from preoperative stage to 5 years after surgery by image-processing software specifically designed for navigation and visualization of multimodality and multidimensional images. Consecutive changes of albumin and body mass index (BMI) as related to general nutritional status were compared between the PG and PJ groups. To evaluate the incidence and risk factors of NODM following PD, subgroup analysis was performed in 88 patients who did not have diabetes preoperatively.

Results: Most patient demographics were not significantly different between the PG group ( $\mathrm{n}=45)$ and PJ group ( $\mathrm{n}=70)$. There was no significant difference in volume reduction between the groups from postoperative 1 month to 5 years (PG group $-18.21 \pm 14.66 \mathrm{~mL}$ versus PJ group $-14.43 \pm 13.05 \mathrm{~mL}, \mathrm{P}=0.209$ ). But there was a significant difference in increased pancreatic duct size between the groups from postoperative 1 month to 5 years (PG group $1.66 \pm 2.20 \mathrm{~mm}$ versus $\mathrm{PJ}$ group $0.54 \pm 1.54 \mathrm{~mm}, \mathrm{P}=0.007$ ). There was no significant difference in the increase of total serum albumin between the groups for 5 years after surgery (PG group $0.51 \pm 0.47 \mathrm{~g} / \mathrm{dL}, 14.3 \%$ versus $\mathrm{PJ}$ group $0.42 \pm 0.60 \mathrm{~g} / \mathrm{dL}, 11.3 \%, \mathrm{P}=0.437$ ). There was also no significant difference in BMI decrease between the groups ( $\mathrm{PG}$ group $-1.13 \pm 3.12,-4.9 \%$ versus $\mathrm{PJ}$ group $-1.97 \pm 2.01,-8.7 \%, \mathrm{P}=0.206)$. On the whole, NODM was diagnosed in 19 patients out of the 88 patients (21.6\%) who did not have DM preoperatively. The incidence of NODM was not significantly different between the groups (PG group 21.6\% versus PJ group 21.5\%, P=0.995). In addition, pancreaticoenterostomy was not an independent risk factor for NODM by logistic regression analysis (odds ratio, 0.997, 95\% CI: 0.356-0.2.788, $\mathrm{P}=0.995)$. No other risk factors for NODM were found.

Conclusions: PG and PJ following PD induced similar pancreatic volume reduction during long-term followup. There was no difference in general nutritional status or incidence of NODM between the groups after PD. 
Keywords: Pancreaticojejunostomy; pancreaticoduodenectomy (PD); nutritional status; diabetes mellitus

Submitted Jan 23, 2019. Accepted for publication Apr 16, 2019.

doi: $10.21037 / \mathrm{hbsn} .2019 .04 .18$

View this article at: http://dx.doi.org/10.21037/hbsn.2019.04.18

\section{Introduction}

Pancreaticoduodenectomy (PD) has been a popular and standardized surgical procedure for treatment of pancreas head and periampullary lesions for several decades $(1,2)$. However, PD is widely known to cause many kinds of endocrine and exocrine deteriorations, because a wide range of pancreatic resections may be included and a substantial structural change after reconstruction is inevitable in the course of an operation. It may also lead to various complications after surgery. Among them, one of the most serious is postoperative pancreatic fistula (POPF). Therefore, pancreaticoenterostomy is the point of interest for the pancreas surgeons doing PD. Pancreatogastrostomy (PG) and pancreatojejunostomy (PJ) are the most commonly used anastomosis methods for pancreaticoenterostomy during PD $(3,4)$.

There have been some studies to compare the surgical results of these two types of pancreaticoenterostomy. They showed that both anastomosis methods are comparable for early postoperative complications, including pancreatic fistula (5-7). As patient survival after PD has increased for several decades, interest in nutrition and new-onset diabetes has been growing. Especially postoperative diabetes has become a notable issue because it has a significant influence on a patient's quality of life. For these reasons, several studies have been done to evaluate nutritional status and new-onset diabetes mellitus (NODM) after PD (8-10). However, few studies have assessed the difference in nutritional status and incidence of NODM between PG and PJ $(10,11)$. In addition, although it is already known that remnant pancreatic volume after PD decreases as time goes on, the differences of volume reduction in a pancreatic remnant between PG and PJ for long-term follow-up are unknown $(9,12)$.

This study aimed to compare serial pancreatic volume changes in pancreatic remnants between PG and PJ after $\mathrm{PD}$ and to evaluate the difference in nutritional status and incidence of NODM between the two methods.

\section{Methods}

\section{Patients}

From January 2001 to December 2014, 422 patients underwent PD for various types of disease, including benign and malignant lesions, at Seoul St. Mary's Hospital, Seoul, Korea. We excluded 282 patients who had died within 3 years after surgery. Also, we excluded four patients who had postoperative pancreatic fistula (POPF) grade $\mathrm{C}$ according to the 2016 International Study Group of Pancreatic Surgery (ISGPS) criteria (13). We also excluded 17 patients who had grade III or IV complications according to the Clavien-Dindo classification (14). Although grade III complication had no influence on postoperative pancreatic volume change and NODM, we contemplated that it can influence postoperative nutritional status. Four patients who had combined resection of other organs, such as the liver and colon, were also excluded. Finally, 115 patients who had survived for more than 3 years after PD and had no serious complications were enrolled in this study. To evaluate the risk factors of NODM following PD, subgroup analysis was also performed. A total of 88 patients without preoperative DM were enrolled in subgroup analysis (Figure 1). Of the enrolled patients, 72 had been diagnosed with a malignancy. Among them, 17 patients were treated with adjuvant chemotherapy, and six patients were diagnosed with recurrence during the follow-up period. Two patients had a local recurrence at the pancreatic remnant and around the superior mesenteric artery. In four patients, tumors recurred at liver, peritoneum, and rib. All the recurrent patients survived for 5 years or more after surgery.

The patients diagnosed with malignant disease had been followed after discharge at the outpatient clinic with laboratory tests and imaging studies (CT or MRI) every 3 months for 2 years after surgery and every 6 months after that until 5 years after surgery. The patients diagnosed with benign disease had been followed after discharge 


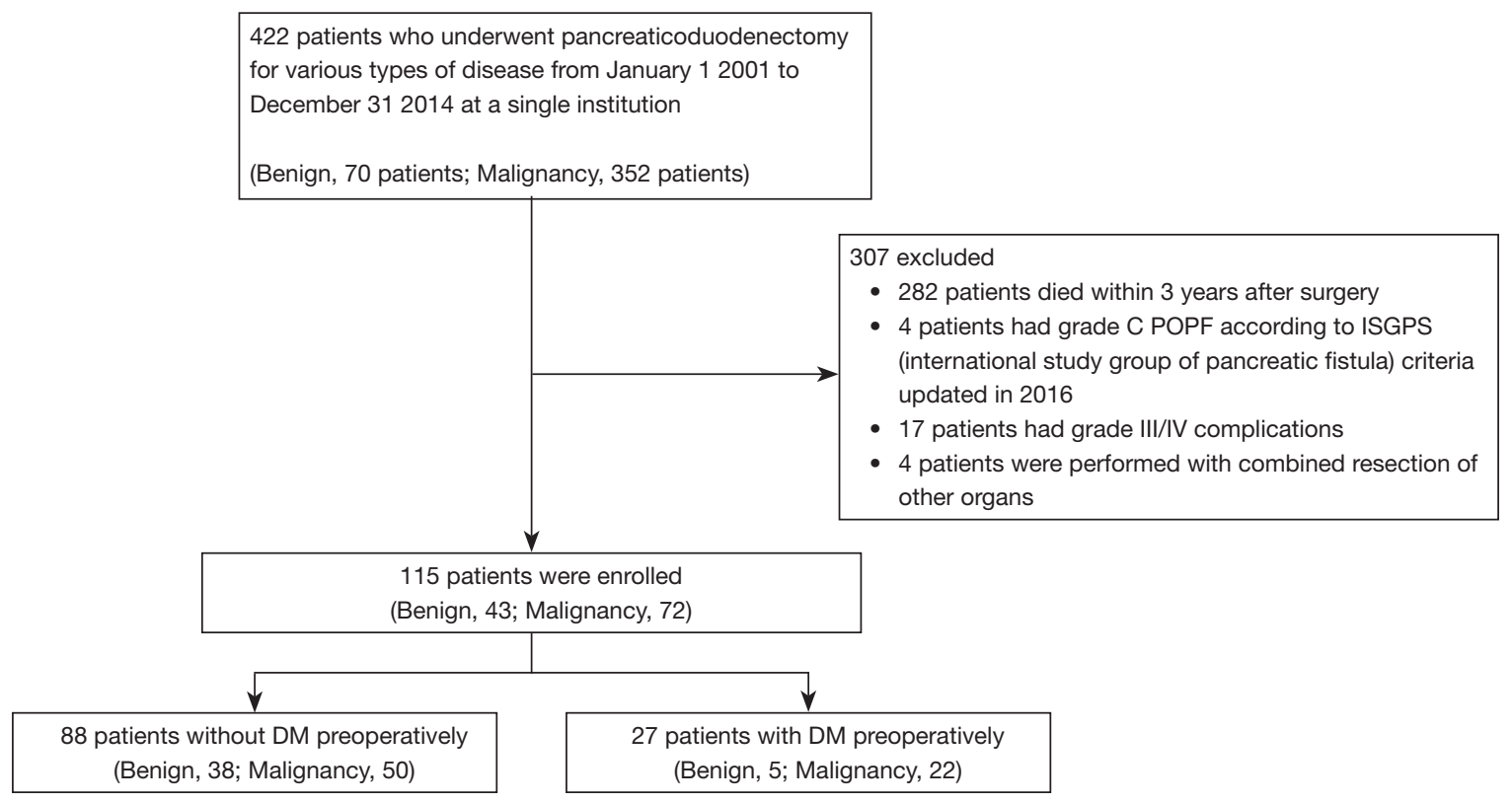

Figure 1 Flow diagram of patient enrollment.

with laboratory tests and imaging studies every 6 months for 2 years after surgery and once a year after that until 5 years.

All data of enrolled patients including imaging data were obtained from the electronic medical records system of our institution. The Institutional Review Board of our center approved the study design (IRB: KC18RCSI0675).

\section{Definitions}

In this study, the diagnostic criteria of NODM were, in patients who had never been diagnosed with diabetes:

(I) When there was either fasting plasma glucose of $126 \mathrm{mg} / \mathrm{dL}$ or higher and HbA1c of $6.5 \%$ or higher;

(II) When there was two-hour plasma glucose of $200 \mathrm{mg} / \mathrm{dL}$ or higher during an oral glucose tolerance test;

(III) When DM medication, including oral hypoglycemic agent or insulin, was started at any time postoperatively.

POPF was defined and graded by an international study group of pancreatic fistula (ISGPF) criteria updated in 2016 (13).

Postoperative complications were defined and graded by Clavien-Dindo classification (14).

Postoperative pancreatic duct dilatation (PDD) was defined as a doubling (or more) of the pancreatic duct diameter at 3 months postoperatively, following a previously reported study (15). Pancreatic duct size was calculated precisely by image-processing software $\left(\mathrm{OsiriX}^{\circledR}\right)$.

After PD, we started oral pancreatic enzyme replacement therapy (Norzyme ${ }^{\circledR}$; Nordmark Arzneimittel GmBH \& Co. $\mathrm{KG}$, Uetersen, Germany) in all patients who had undergone PD and had started an oral diet, and stopped the therapy 3 months after surgery. We selected the patients who experienced foul-smelling, greasy, and floating stools during regular follow-up. Among them, patients who complained about feeling uncomfortable in social life were diagnosed with steatorrhea.

\section{Pancreaticoenterostomy (PG versus P7)}

In our institution, more than five surgeons have been performing PD and pancreaticoenterostomy method was basically decided according to the operator's preference. Otherwise, some of them tended to choose PG rather than PJ for patients with extremely small pancreatic duct and bulky pancreas with a soft texture. For the PG, the pancreatic remnant was anastomosed to the posterior antrum wall of the stomach. After sufficient mobilization of the pancreatic stump, the stump was inserted into the posterior antrum wall of the stomach. At that time, the duct of Wirsung was identified and cannulated with a short stent 


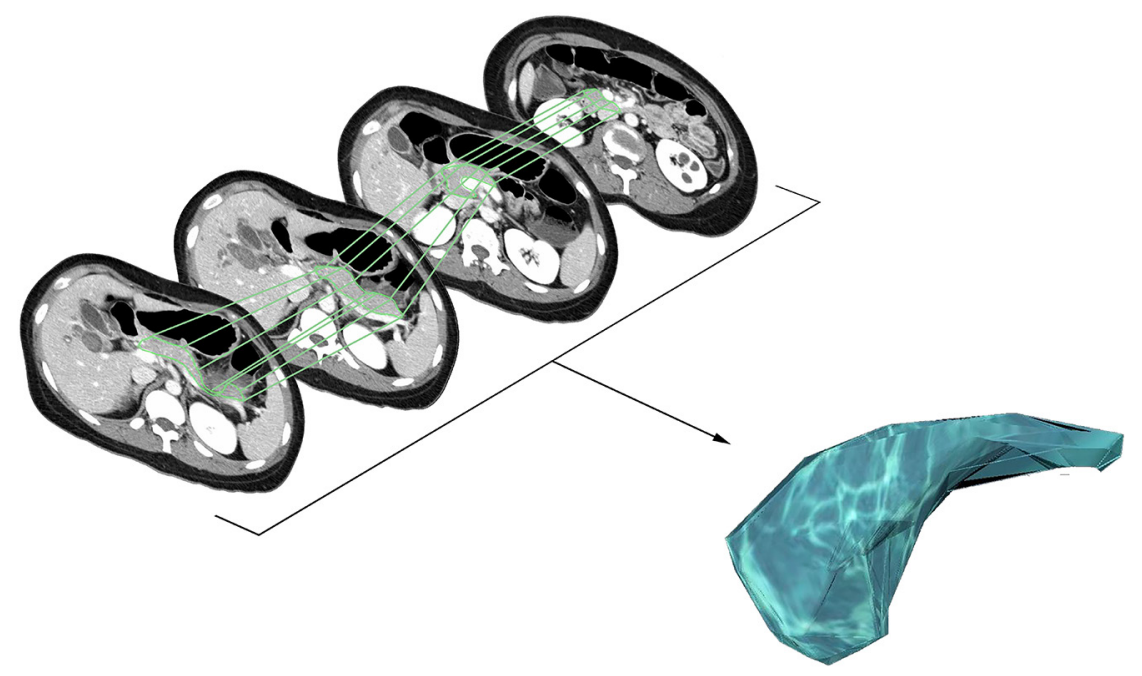

Figure 2 The method for calculating pancreas volume. The pancreas parenchyma was outlined manually at every section as regions of interest (ROI, the area inside the green line). ROI volume was calculated by using imaging software, OsiriX. Tumors, cystic lesions, vessels, dilated bile duct, and dilated pancreatic duct were excluded from the calculation of the volume.

tube to avoid it's being sutured during the anastomosing of the pancreatic remnant to the stomach. PG was fixed using single-layer 3-0 Vicryl interrupted sutures, and the cut surface of the pancreatic stump was also closed using 3-0 Vicryl interrupted stitches to prevent bleeding. For the PJ, the pancreatic remnant was anastomosed to the anti-mesenteric border of the jejunum using a duct-tomucosa technique with an internal stent. The interrupted sutures between the pancreatic parenchyma and the jejunal seromuscular were placed in the outer layer using absorbable Vicryl \#3-0 sutures. A small hole compatible with the caliber of the pancreatic duct was made in the jejunal wall using electrocautery. The pancreatic duct was then directly sutured to the jejunal mucosa with a short internal stent by absorbable sutures (Monosyn \#4-0 or \#5-0) using four to six interrupted stitches.

\section{Assessment of pancreas volume, pancreatic duct size, and texture}

To measure the exact pancreas volume and pancreatic duct size, we collected computed tomography (CT) or magnetic resonance imaging (MRI) preoperatively and postoperatively at 1 month, 6 months, 1 year, 3 years, and 5 years. Three-dimensional volumetry was performed using imaging software named OsiriX (OsiriX 9.5, Pixmeo SARL, Geneva, Switzerland). Using this program, we outlined the pancreas manually at every section showing pancreas parenchyma as regions of interest (ROI, the area inside the green line). At that time, tumors, cystic lesions, vessels, dilated bile duct and dilated pancreatic duct were excluded from ROI. Then the ROI volume was calculated automatically (Figure 2). The resected pancreas volume was calculated by subtracting the remnant pancreas volume at 1 month postoperative from the preoperative whole pancreas volume. The resected volume ratio (RVR) was presented by a percentage. It was defined as the ratio of resected pancreas volume to the preoperative entire pancreas volume. Using the same software, pancreatic duct size was also measured. We examined the maximal diameter of the pancreatic duct at each imaging. There is no absolutely objective method for evaluating the softness of the pancreas. Only a few radiological studies have addressed the issue of how pancreas texture can be detected and quantified (16). So we classified pancreas texture by using medical records describing intraoperative findings. The texture was classified to soft or hard.

\section{Statistical analysis}

Numerical data are presented as means and standard deviations (or standard errors). Continuous variables were analyzed using the independent $t$-test, one-way analysis of variance, or the Kruskal-Wallis test, as appropriate. 
Proportions were tested using Pearson's chi-square test or Fisher's exact test. Repeated-measure ANOVA and a linear mixed model were used to analyze the interaction between two groups that had repeated measured data. A logistic regression model was used for univariate and multivariate analyses. The statistical analysis was performed using SPSS software (ver. 24.0; SPSS, Inc., Chicago, IL, USA). A $\mathrm{P}<0.05$ was considered significant.

\section{Results}

\section{Demographics}

In all study populations, the mean follow-up period was 100.2 months (median 88.0 months, range 42.0-201.0 months). Patient characteristics in the PG group $(n=45)$ and PJ group $(n=70)$ are shown in Table 1 . Among the 115 patients, benign diseases were diagnosed in 43 patients. Intraductal papillary mucinous neoplasm (IPMN) ( $\mathrm{n}=14)$ was most common, followed by pancreatitis $(n=5)$, neuroendocrine tumor (NET) $(n=4)$, and serous cystic neoplasm $(\mathrm{SCN})(\mathrm{n}=3)$. Malignant diseases were diagnosed in 72 patients. Ampulla of Vater (AoV) cancer $(n=40)$ was most common, followed by bile duct cancer $(n=20)$, pancreas cancer $(n=6)$, duodenal cancer $(n=3)$, and neuroendocrine cancer $(\mathrm{n}=1)$.

Surgical procedures included conventional PD $(n=32)$ and pylorus-preserving PD (PPPD) ( $\mathrm{n}=83)$. There were 24 patients $(75 \%)$ in the $\mathrm{PG}$ group and 42 patients $(63.6 \%)$ in the PJ group who had undergone pylorus-preserving surgery. There was no significant difference in the ratio of pylorus-preserving surgery between the two groups $(\mathrm{P}=0.261)$. Age, sex, body mass index (BMI), American Society of Anesthesiologists (ASA) score, preoperative symptoms, operation time, blood loss, pancreatic duct size at preoperative imaging, proportion of benign and malignant diseases, and incidence of POPF (biochemical leak and grade B), postoperative complications (grades I and II), and preoperative diabetes were not significantly different between the two groups. The PG group showed a significantly higher rate of laparoscopic approach than did the PJ group (PG, n=13/45, 28.9\%; PJ, n=4/70, 5.7\%; $\mathrm{P}=0.001)$. Compared to the $\mathrm{PJ}$ group, the number of soft pancreases was significantly higher in the $\mathrm{PG}$ group (PG, $\mathrm{n}=33 / 45,73.3 \%$; PJ, $\mathrm{n}=33 / 70,47.1 \%$; $\mathrm{P}=0.006$ ), and hospital stay was significantly shorter in the $\mathrm{PG}$ group than in the PJ group (PG, 16.11 \pm 6.27 days; PJ, 19.31 \pm 6.00 days; $\mathrm{P}=0.007)$.

\section{Pancreas volume and pancreatic duct size after PD}

We compared the resected volume of the pancreas and a RVR between the PG and PJ groups. There was no significant difference in resected volume (PG group, $23.07 \pm 21.64 \mathrm{~mL}$, versus PJ group, 22.56 $\pm 18.30 \mathrm{~mL}$, $\mathrm{P}=0.901$ ) or RVR (PG group, $35.18 \% \pm 24.38 \%$, versus PJ group, $41.25 \% \pm 19.79 \%, \mathrm{P}=0.225)$ between the groups (Table 1).

We also analyzed consecutive changes of pancreas volume and pancreatic duct size after PD during the followup period. For the whole follow-up period, there was no significant difference in volume reduction between the groups, from postoperative 1 month to 5 years (PG group, $-18.21 \pm 14.66 \mathrm{~mL}$, versus $\mathrm{PJ}$ group, $-14.43 \pm 13.05 \mathrm{~mL}$, $\mathrm{P}=0.209)$. However, there was a clear difference in the details of the change. In both groups, pancreas volume had decreased rapidly in the early stages after surgery, postoperative 1 to 6 months. However, the PG group showed much more volume reduction than did the PJ group in this period. Six months after surgery, both groups showed gradual volume reduction until 5 years after surgery. In this period, the change in volume over time was not statistically significant in the $\mathrm{PG}$ group $(\mathrm{P}=0.347)$. On the other hand, it was statistically significant in the $\mathrm{PJ}$ group $(\mathrm{P}=0.034)$ (Figure 3A).

Preoperatively, there was no difference in pancreatic duct size between the groups $(\mathrm{P}=0.239)$. However, starting 1 month after surgery, pancreatic duct size in the PG group steadily increased until 5 years after surgery, which was statistically significant $(\mathrm{P}=0.032)$. On the other hand, pancreatic duct size in the PJ group was similar from 1 month to 5 years after surgery $(\mathrm{P}=0.202)$. Finally, there was a significant difference in pancreatic duct size increase between the groups from postoperative 1 month to 5 years (PG group, $1.66 \pm 2.20 \mathrm{~mm}$, versus PJ group, 0.54 $\pm 1.54 \mathrm{~mm}, \mathrm{P}=0.007$ ) (Figure $3 B$ ).

\section{General nutritional status and incidence of NODM}

We analyzed consecutive changes of parameters related to general nutritional status. Mean preoperative serum albumin level was $3.56 \pm 0.47$ and $3.72 \pm 0.48 \mathrm{~g} / \mathrm{dL}$ in the PG group and the PJ group, respectively $(\mathrm{P}=0.123)$. In our institution, reference normal albumin ranged from 3.5 to $5.2 \mathrm{~g} / \mathrm{dL}$. Serum albumin level started to decrease postoperatively, reached $2.96 \pm 0.36 \mathrm{~g} / \mathrm{dL}(-16.9 \%)$ and $3.01 \pm 0.57 \mathrm{~g} / \mathrm{dL}(-19.1 \%)$ at postoperative day 5 to $7 \mathrm{in}$ 
Table 1 Demographics according to anastomosis method.

\begin{tabular}{|c|c|c|c|}
\hline Demographics & $P G(n=45)$ & PJ $(n=70)$ & $P$ value \\
\hline Sex & & & 0.777 \\
\hline Male & $25(55.6)$ & $37(52.9)$ & \\
\hline Female & $20(44.4)$ & $33(47.1)$ & \\
\hline ASA score & & & 0.298 \\
\hline 1,2 & 42 (93.3) & $69(98.6)$ & \\
\hline 3 & $3(6.7)$ & $1(1.4)$ & \\
\hline Preoperative symptom & & & 0.074 \\
\hline Operation time (min) & $372.33 \pm 135.43$ & $376.64 \pm 90.45$ & 0.851 \\
\hline Blood loss (mL) & $748.41 \pm 714.82$ & $932.61 \pm 933.29$ & 0.298 \\
\hline Operation type & & & 0.001 \\
\hline Open PPPD/PD & $24 / 8(71.1)$ & 42/24 (94.3) & \\
\hline Laparoscopic PPPD & $13(28.9)$ & $4(5.7)$ & \\
\hline Pancreatic duct size (mm) & & & 0.239 \\
\hline$\leq 3$ & $26(66.7)$ & $47(77.0)$ & \\
\hline$>3, \leq 10$ & $12(30.8)$ & $14(23.0)$ & \\
\hline Benign & $19(42.2)$ & $24(34.3)$ & \\
\hline SCN & 0 & 3 & \\
\hline Pancreatitis & 0 & 5 & \\
\hline IPMN & 10 & 4 & \\
\hline NET & 3 & 1 & \\
\hline Others & 6 & 11 & \\
\hline Malignancy & $26(57.8)$ & $46(65.7)$ & \\
\hline Bile duct cancer & 8 & 12 & \\
\hline Pancreas cancer & 1 & 5 & \\
\hline AoV cancer & 16 & 24 & \\
\hline Duodenal cancer & 0 & 3 & \\
\hline
\end{tabular}

Table 1 (continued) 
Table 1 (continued)

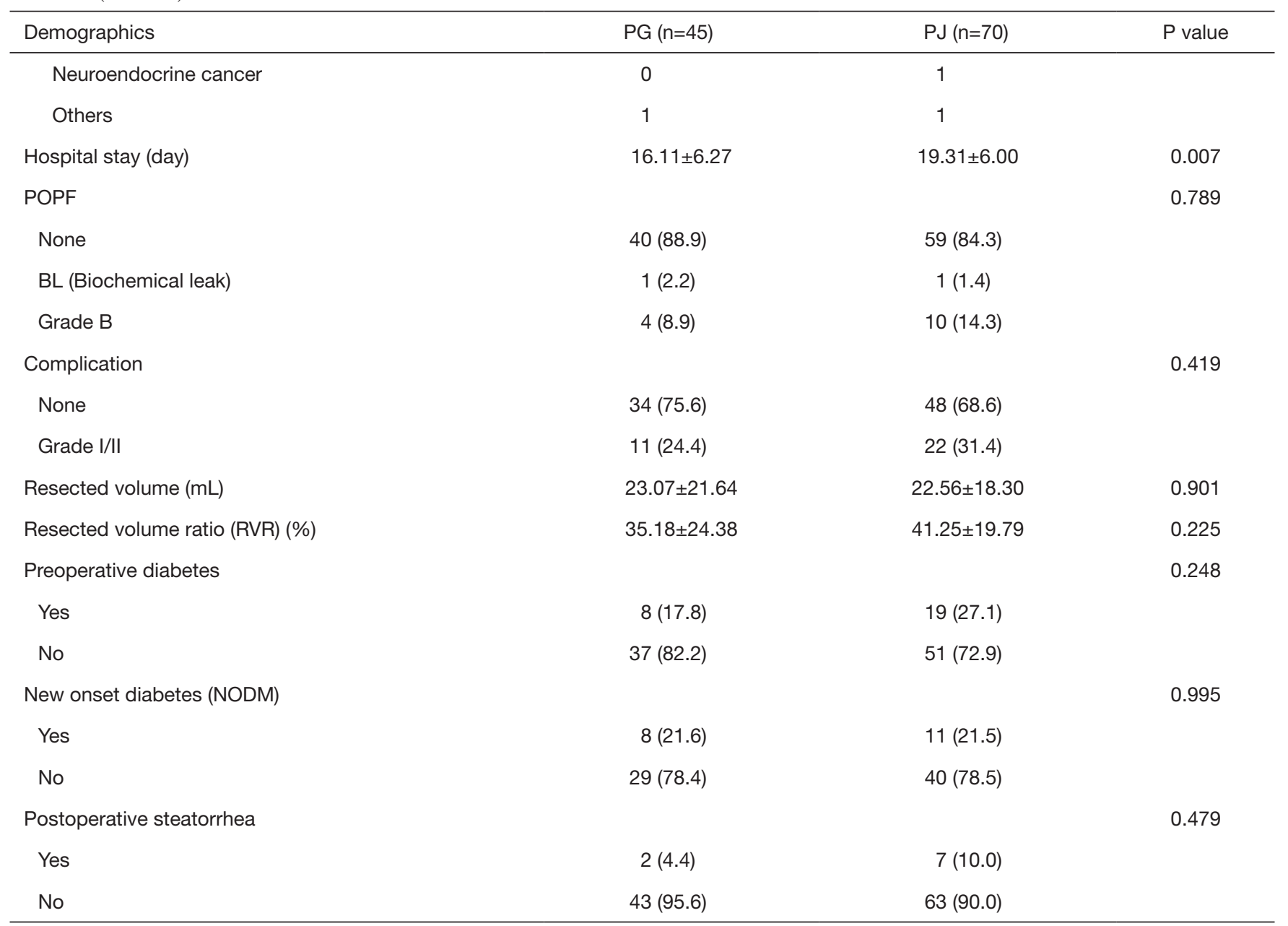

Values are presented in mean \pm standard deviation, or $n$ (\%). PG, pancreaticogastrostomy; PJ, pancreaticojejunostomy; BMI, body mass index; ASA, American society of anesthesiologists; PPPD, pylorus-preserving pancreaticoduodenectomy; PD, pancreaticoduodenectomy; SCN, serous cystic neoplasm; IPMN, intraductal papillary mucinous neoplasm; NET, neuroendocrine tumor; AoV, ampulla of Vater; POPF, postoperative pancreatic fistula, POPF was defined and graded by ISGPS (international study group of pancreatic fistula) criteria updated in 2016 (13); BL, biochemical leak; complications was defined and graded to Clavien-Dindo classification (14); resected volume ratio (RVR) was defined as the resected pancreas volume divided by the preoperative pancreas volume.

both groups $(\mathrm{P}=0.215)$. It recovered to a preoperative level within 2 weeks after surgery. Then it rapidly increased and reached over $4.0 \mathrm{~g} / \mathrm{dL}$ in both groups at 6 months after surgery. It then gradually increased during the 5 years after surgery. At every examination point, there were no significant differences in serum albumin level between the groups. There was also no significant difference in total serum albumin level change between the groups for 5 years after surgery (PG group, $0.51 \pm 0.47 \mathrm{~g} / \mathrm{dL}, 14.3 \%$, versus $\mathrm{PJ}$ group, $0.42 \pm 0.60 \mathrm{~g} / \mathrm{dL}, 11.3 \%, \mathrm{P}=0.437)$.

Preoperative BMI was not different between the groups (PG group, 22.97 $\pm 3.41 \mathrm{~mm}$, versus $\mathrm{PJ}$ group, $22.72 \pm 3.46 \mathrm{~mm}, \mathrm{P}=0.701)$. The most significant decrease in BMI occurred 6 months after surgery, by -1.01 (4.4\%) in the PG group and by $-1.37(6.0 \%)$ in the PJ group $(\mathrm{P}=0.125)$. Since then, up to 5 years after surgery, no major changes were seen in BMI. At all examination points, there were no significant differences in BMI between the PG and $\mathrm{PJ}$ groups. There was also no significant difference in BMI decrease between the groups during the 5 years after surgery (PG group, $-1.13 \pm 3.12,-4.9 \%$, versus PJ group, $-1.97 \pm 2.01$, $-8.7 \%, \mathrm{P}=0.206$ ) (Figure $4 A, B)$. Two patients $(4.4 \%)$ in 


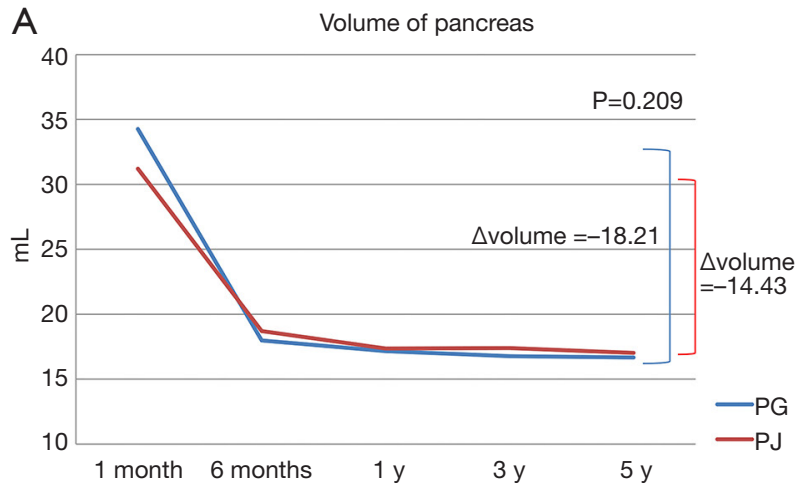

\begin{tabular}{|c|c|c|c|c|c|}
\hline $\begin{array}{c}\text { Volume } \\
(\text { mean } \pm \text { SE) }\end{array}$ & 6 months & $1 y$ & $3 y$ & $5 y$ & P value \\
\hline PG $(n=45)$ & $17.98 \pm 1.18$ & $17.16 \pm 1.27$ & $16.77 \pm 1.34$ & $16.66 \pm 1.43$ & 0.347 \\
\hline PJ $(\mathrm{N}=70)$ & $18.69 \pm 1.44$ & $17.35 \pm 1.42$ & $17.38 \pm 1.49$ & $17.02 \pm 1.62$ & $\mathbf{0 . 0 3 4}$ \\
\hline
\end{tabular}

B

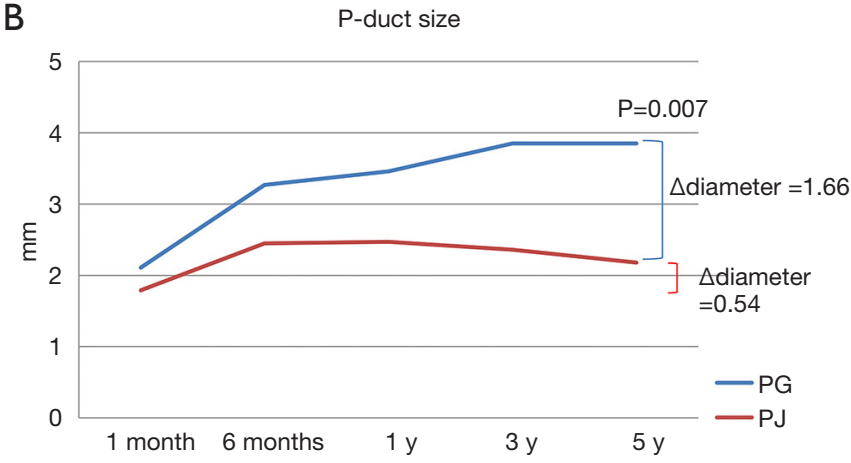

\begin{tabular}{|c|c|c|c|c|c|}
\hline $\begin{array}{c}\text { Volume } \\
(\text { mean } \pm \text { SE) }\end{array}$ & 6 months & $1 y$ & $3 y$ & $5 y$ & P value \\
\hline$P G(n=45)$ & $3.57 \pm 0.32$ & $3.88 \pm 0.35$ & $4.09 \pm 0.40$ & $3.99 \pm 0.34$ & 0.032 \\
\hline$P J(N=70)$ & $2.83 \pm 0.15$ & $2.76 \pm 0.15$ & $2.59 \pm 0.12$ & $2.64 \pm 0.13$ & 0.202 \\
\hline
\end{tabular}

Figure 3 Consecutive pancreas volume and pancreatic duct size after pancreaticoduodenectomy according to pancreas reconstruction method: (A) volume of the pancreas; (B) pancreatic duct size. PG, pancreatogastrostomy; PJ, pancreatojejunostomy.

A

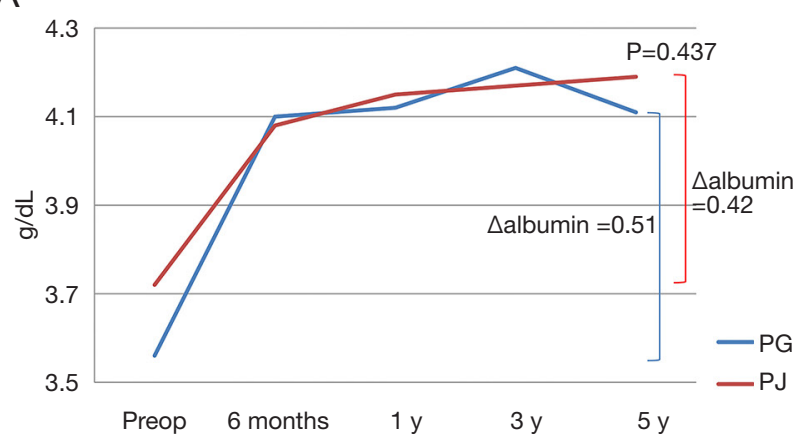

B

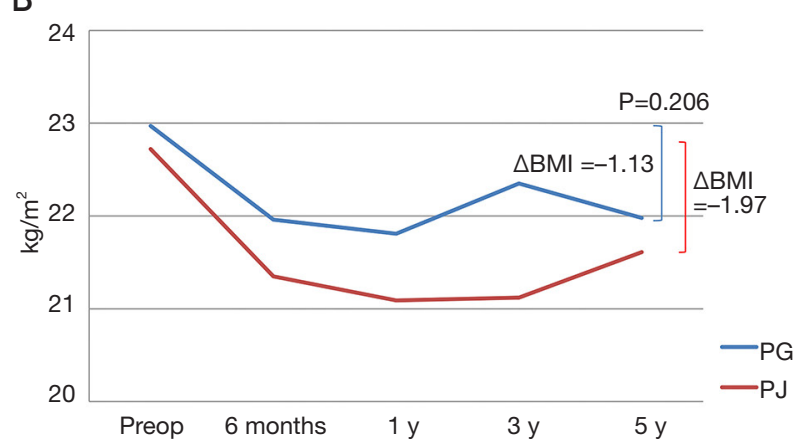

Figure 4 Changes in parameters related to general nutritional status according to the time after surgery: (A) serum albumin; (B) BMI (body mass index). PG, pancreatogastrostomy; PJ, pancreatojejunostomy.

the PG group and seven patients $(10.0 \%)$ in the PJ group experienced postoperative steatorrhea during the 5 years after surgery, but there was no significant difference between the groups $(\mathrm{P}=0.479)$ (Table 1).

In the analysis of 88 patients without preoperative DM, 19 patients $(21.6 \%)$ had been diagnosed with NODM during the follow-up period after PD. There was no significant difference in the incidence of NODM between the groups (PG group 21.6\% versus PJ group 21.5\%, $\mathrm{P}=0.995$ ) (Table 1). We investigated the time of diagnosis of NODM to evaluate the incidence of NODM according to the period; there was a heterogeneous pattern in both groups. There was no significant difference in the rate of
NODM according to the period between the PG and PJ groups at every examination point (Figure 5). The logistic regression model analysis to evaluate the risk factors for NODM showed that pancreaticoenterostomy was not an independent risk factor for NODM (odds ratio 0.997; 95\% CI: $0.356-2.788, \mathrm{P}=0.995)$. In addition, age, sex, BMI, resection type (laparoscopic versus open), the whole volume of the pancreas, and the overall volume of pancreas/body weight ratio were not significant risk factors for NODM after PD. Diagnosis (malignant versus benign), pancreas texture (soft versus hard), adjuvant chemotherapy, tumor recurrence, and postoperative PDD were not significant risk factors either (Table 2). 


\section{Discussion}

In this study, we found the following. First, after PD, the pancreatic remnant showed significant rapid volume reduction until postoperative 6 months and after that stabilized. There was no significant difference in overall volume reduction of the pancreatic remnant between the PG and PJ groups for 5 years after PD. However, the pancreatic duct in the PG group was significantly more

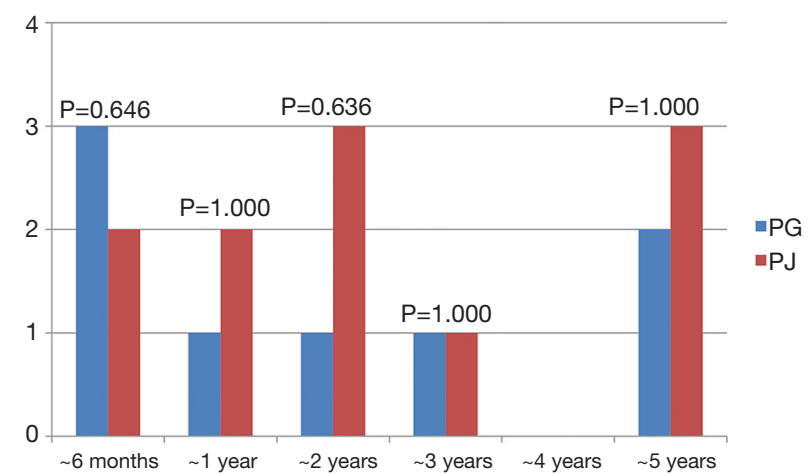

Figure 5 The incidence of new-onset DM (NODM) according to the time after surgery. PG, pancreatogastrostomy; PJ, pancreatojejunostomy. dilated than in the PJ group during the same period.

Second, nutritional status represented by albumin and BMI was not different between the groups during the 5 years after surgery. Serum albumin level decreased postoperatively and recovered within two weeks. After then, it tended to increase steadily. BMI showed a steep decrease within postoperative 6 months and then remained at a similar level until 5 years after surgery.

Third, there was no significant difference in the incidence of NODM between the PG and PJ groups. The logistic regression analysis to evaluate risk factors for NODM showed that pancreaticoenterostomy was not an independent risk factor. We also could not find any other risk factors affecting NODM in the cohort of this study.

There have been several studies to calculate the pancreatic volume before and after PD. Some studies investigated pancreatic volume after PD to evaluate the relationship between the resected amount of pancreas and NODM $(8,9,12)$. Iizawa et al. also calculated pancreatic mass during 3 years after PD to evaluate the relationship between remnant pancreatic volume and pancreatic endocrine and exocrine dysfunction. In that study, pancreatic volume rapidly decreased until 6 months after PD and after that stabilized until 3 years after surgery. Our results showed a

Table 2 Risk factors for new-onset DM (NODM) in patients after pancreaticoduodenectomy

\begin{tabular}{|c|c|c|}
\hline Risk factor & \multicolumn{2}{|c|}{ Univariate analysis of NODM } \\
\hline Age (years) & $1.016(0.975-1.060)$ & 0.451 \\
\hline Sex (female) & $0.825(0.298-2.281)$ & 0.711 \\
\hline BMI $\left(\mathrm{kg} / \mathrm{m}^{2}\right)$ & $1.040(0.905-1.196)$ & 0.577 \\
\hline Whole volume of pancreas (mL) & $0.975(0.948-1.003)$ & 0.081 \\
\hline Whole volume/body weight (mL/kg) & $0.178(0.029-1.078)$ & 0.060 \\
\hline Resected volume (mL) & $0.964(0.924-1.005)$ & 0.081 \\
\hline Resected volume ratio (\%) & $0.321(0.023-4.483)$ & 0.398 \\
\hline Pancreas texture (soft) & $1.571(0.534-4.621)$ & 0.412 \\
\hline Adjuvant chemotherapy & $1.149(0.327-4.038)$ & 0.829 \\
\hline Tumor recurrence (local and/or distant) & $0.711(0.078-6.481)$ & 0.762 \\
\hline Pancreatic duct dilatation & $0.394(0.079-1.965)$ & 0.256 \\
\hline
\end{tabular}

$\mathrm{HR}$, hazard ratio; $\mathrm{Cl}$, confidence interval; $\mathrm{PJ}$, pancreaticojejunostomy. 
similar pattern of decrease in pancreatic volume for 5 years after PD. They speculated that the early postoperative shrinkage of the pancreatic remnant might be caused by the decreased blood flow after transection of the pancreas (9). In addition, recovery of edematous change of the pancreas caused by complex surgery that takes a long time could affect the volume change within 6 months after surgery.

The difference in reduction of pancreatic volume between PG and PJ after PD during a long-term follow-up has been almost un known before now. We could find only one similar study. Tomimaru et al. compared the change of parenchymal thickness between PG and PJ preoperatively and 2 years after PD. They found a significant thickness reduction of $46.0 \%$ in the PG group and $22.4 \%$ in the PJ group. There was a statistically significant difference in reduction rate between the groups. In this study, there was more volume reduction in the PG group than in the PJ group during the 6 months after surgery. On the other hand, there was no significant difference between the PG and PJ groups in the overall volume reduction of the pancreatic remnant for 5 years after PD. This is a conclusion very different from our analysis results. We think that this discrepancy may have resulted from differences in the follow-up period and method for calculating pancreas volume. Although measurement of parenchymal thickness can be used to estimate the volume of the pancreatic remnant, it is not an exact volume calculation. Therefore, the two studies cannot be compared equally.

In contrast to the situation with $\mathrm{PJ}$ anastomosis, the pancreatic duct in PG anastomosis is repeatedly exposed to food material mixed with gastric juice. That environment can facilitate recurrent pancreatic duct obstruction following PDD for a long time. Our results showed that pancreatic duct size increased significantly more in the PG group than in the PJ group for 5 years after surgery. Tomimaru et al. reported similar results. They compared the change of the main pancreatic duct (MPD) diameter between the PG and PJ groups preoperatively and at 2 years after PD. Their study showed that MPD diameter significantly increased more in the PG group than in the PJ group at 2 years after surgery (17).

General nutritional status after PD according to the analysis of albumin level and BMI seems to be similar between the PG and PJ groups for long-term followup. The PD procedure includes a wide range of resection of digestive organs and a substantial reconstruction, and it leads to many kinds of endocrine and exocrine deteriorations after PD. For that reason, serum albumin level decreased temporarily within 2 weeks after surgery, but then gradually increased in this study. The BMI declined rapidly until 6 months after surgery, then maintained the decreased level, although it was within normal range. Patients who underwent PD tended to reduce their food intake themselves after surgery, which could also affect the maintenance of the decreased BMI level. Some previous studies reported that the incidence of postoperative steatorrhea after PD was higher in the PG group than in the PJ group $(10,11)$. Bock et al. reported that $52.8 \%$ of patients who had undergone PD with PG experienced steatorrhea postoperatively (18). However, in our study, only 2 patients (4.4\%) in the PG group and 7 patients $(10.0 \%)$ in the PJ group experienced postoperative steatorrhea during the 5 years after surgery. This discrepancy seemed to result from the definition of steatorrhea in this study. We diagnosed steatorrhea if the patient's symptoms caused uncomfortable feelings in social life without evaluating fecal elastase. In addition, the difference between the studies in the amount and duration of use of enzyme substitutes could affect the incidence rate of steatorrhea.

Given increasing patient survival after $\mathrm{PD}$, postoperative diabetes has become a more critical issue (19). In our study, $21.6 \%$ of all patients without preoperative DM had been diagnosed with NODM during the follow-up period after PD. There was no significant difference in the incidence of NODM between the PG and PJ groups. Previous studies presented age, BMI, diagnosis (PDAC: pancreatic ductal adenocarcinoma), PDD, and pancreatic volume reduction rate 6 months after $\mathrm{PD}$ as independent risk factors for NODM after PD $(8,9,12,18)$. However, we could not find any risk factors affecting NODM after $\mathrm{PD}$, including previously described factors, in our research cohort. If the PDD greatly affects the constriction of pancreatic parenchyma, it might become one of the determinants of NODM. However, that was not the case in our study. Otherwise, as can be seen in this study, serial changes in pancreatic duct did not have a clear correlation with pancreatic volume. Hence, we hypothesized that postoperative PDD did not trigger NODM.

In this study, the PG group had a significantly higher rate of laparoscopic approach than did the PJ group, because PG using mini-laparotomy was mainly performed through laparoscopic PD from 2009 to 2013 in our institution. Hospital stay was significantly shorter in the PG group than in the PJ group, perhaps because of the higher rate of laparoscopic approach in the PG group. We tended to choose PG rather than PJ for patients who had a bulky 
pancreas with a tiny pancreatic duct and a soft texture. As a result, pancreas texture was significantly softer in the PG group than in the PJ group.

This study is meaningful in that it is one of the rare studies that serially evaluate pancreatic volume change between the PG and PJ groups after PD, and it included more patients than have similar kinds of studies. In addition, we used software $\left(\right.$ OsiriX ${ }^{\circledR}$ ) that has been specifically designed for navigation and visualization of multimodality and multidimensional images to measure pancreatic volume and pancreatic duct size. Using the program, very sophisticated ROI selection was possible. Based on it, the pancreatic amount and pancreatic duct size could be measured more accurately than by the previous manual calculation.

It is still unclear which pancreaticoenterostomy method, of PG or PJ, is superior for the prevention of POPF following PD (20,21). Considering the references above and our research results together, it seemed that there was no significant difference in pancreas volume reduction and incidence of NODM after PD between the groups for long-term follow-up. Depending on these results, the pancreatoenterostomy method can be chosen by the preference of each surgeon. However, each surgical case has a specific condition of disease and anatomy, and each surgeon has different surgical skills. Therefore, we suggest that the pancreatoenterostomy method can be chosen according to the particular situation of the surgical case and the craft and preference of the surgeon.

\section{Acknowledgments}

Funding: None.

\section{Footnote}

Conflicts of Interest: All authors have completed the ICMJE uniform disclosure form (available at https://hbsn. amegroups.com/article/view/10.21037/hbsn.2019.04.18/coif). The authors have no conflicts of interest to declare.

Ethical Statement: The authors are accountable for all aspects of the work in ensuring that questions related to the accuracy or integrity of any part of the work are appropriately investigated and resolved. The study protocol was approved by Institutional Review Board of our center (IRB: KC18RCSI0675).
Open Access Statement: This is an Open Access article distributed in accordance with the Creative Commons Attribution-NonCommercial-NoDerivs 4.0 International License (CC BY-NC-ND 4.0), which permits the noncommercial replication and distribution of the article with the strict proviso that no changes or edits are made and the original work is properly cited (including links to both the formal publication through the relevant DOI and the license). See: https://creativecommons.org/licenses/by-nc$\mathrm{nd} / 4.0 /$.

\section{References}

1. Cameron JL, Pitt HA, Yeo CJ, et al. One hundred and forty-five consecutive pancreaticoduodenectomies without mortality. Ann Surg 1993;217:430-5; discussion 435-8.

2. Yeo CJ, Cameron JL, Sohn TA, et al. Six hundred fifty consecutive pancreaticoduodenectomies in the 1990s: pathology, complications, and outcomes. Ann Surg 1997;226:248-57; discussion 257-60.

3. Wang W, Zhang Z, Gu C, et al. The optimal choice for pancreatic anastomosis after pancreaticoduodenectomy: A network meta-analysis of randomized control trials. Int J Surg 2018;57:111-6.

4. Tewari M, Hazrah P, Kumar V, et al. Options of restorative pancreaticoenteric anastomosis following pancreaticoduodenectomy: a review. Surg Oncol 2010;19:17-26.

5. Icard P, Dubois F. Pancreaticogastrostomy following pancreatoduodenectomy. Ann Surg 1988;207:253-6.

6. Delcore R, Thomas JH, Pierce GE, et al. Pancreatogastrostomy: a safe drainage procedure after pancreatoduodenectomy. Surgery 1990;108:641-5; discussion 645-7.

7. Hallet J, Zih FS, Deobald RG, et al. The impact of pancreaticojejunostomy versus pancreaticogastrostomy reconstruction on pancreatic fistula after pancreaticoduodenectomy: meta-analysis of randomized controlled trials. HPB (Oxford) 2015;17:113-22.

8. Kwon JH, Kim SC, Shim IK, et al. Factors Affecting the Development of Diabetes Mellitus After Pancreatic Resection. Pancreas 2015;44:1296-303.

9. Iizawa $\mathrm{Y}$, Kato H, Kishiwada M, et al. Long-term outcomes after pancreaticoduodenectomy using pairwatch suturing technique: Different roles of pancreatic duct dilatation and remnant pancreatic volume for the development of pancreatic endocrine and exocrine 
dysfunction. Pancreatology 2017;17:814-21.

10. Jang JY, Kim SW, Park SJ, et al. Comparison of the functional outcome after pylorus-preserving pancreatoduodenectomy: pancreatogastrostomy and pancreatojejunostomy. World J Surg 2002;26:366-71.

11. Schmidt U, Simunec D, Piso P, et al. Quality of life and functional long-term outcome after partial pancreatoduodenectomy: pancreatogastrostomy versus pancreatojejunostomy. Ann Surg Oncol 2005;12:467-72.

12. Yun SP, Seo HI, Kim S, et al. Does the pancreatic volume reduction rate using serial computed tomographic volumetry predict new onset diabetes after pancreaticoduodenectomy? Medicine (Baltimore) 2017;96:e6491.

13. Bassi C, Marchegiani G, Dervenis C, et al. The 2016 update of the International Study Group (ISGPS) definition and grading of postoperative pancreatic fistula: 11 Years After. Surgery 2017;161:584-91.

14. Dindo D, Demartines N, Clavien PA. Classification of surgical complications: a new proposal with evaluation in a cohort of 6336 patients and results of a survey. Ann Surg 2004;240:205-13.

15. Tani M, Onishi H, Kinoshita H, et al. The evaluation of duct-to-mucosal pancreaticojejunostomy in pancreaticoduodenectomy. World J Surg 2005;29:76-9.

16. Yardimci S, Kara YB, Tuney D, et al. A Simple Method to Evaluate Whether Pancreas Texture

Cite this article as: Kwak BJ, Choi HJ, You YK, Kim DG, Hong TH. Comparative long-term outcomes for pancreatic volume change, nutritional status, and incidence of new-onset diabetes between pancreatogastrostomy and pancreatojejunostomy after pancreaticoduodenectomy. HepatoBiliary Surg Nutr 2020;9(3):284-295. doi: 10.21037/ hbsn.2019.04.18
Can Be Used to Predict Pancreatic Fistula Risk After Pancreatoduodenectomy. J Gastrointest Surg 2015;19:1625-31.

17. Tomimaru Y, Takeda Y, Kobayashi S, et al. Comparison of postoperative morphological changes in remnant pancreas between pancreaticojejunostomy and pancreaticogastrostomy after pancreaticoduodenectomy. Pancreas 2009;38:203-7.

18. Bock EA, Hurtuk MG, Shoup M, et al. Late complications after pancreaticoduodenectomy with pancreaticogastrostomy. J Gastrointest Surg 2012;16:914-9.

19. Chia CL, Lee AY, Shelat VG, et al. Does diabetes mellitus affect presentation, stage and survival in operable pancreatic cancer? Hepatobiliary Surg Nutr 2016;5:38-42.

20. Cheng Y, Briarava M, Lai M, et al. Pancreaticojejunostomy versus pancreaticogastrostomy reconstruction for the prevention of postoperative pancreatic fistula following pancreaticoduodenectomy. Cochrane Database Syst Rev 2017;9:CD012257.

21. Aroori S, Puneet P, Bramhall SR, et al. Outcomes comparing a pancreaticogastrostomy $(\mathrm{PG})$ and a pancreaticojejunostomy $(\mathrm{PJ})$ after a pancreaticoduodenectomy (PD). HPB (Oxford) 2011;13:723-31. 